\title{
Target annihilation by diffusing particles in inhomogeneous geometries
}

\author{
Davide Cassi \\ Dipartimento di Fisica, Università di Parma, \\ viale Usberti 7/a, 43100 Parma, ITALY
}

(Date textdate; Received text; Revised text; Accepted text; Published text)

\begin{abstract}
The survival probability of immobile targets, annihilated by a population of random walkers on inhomogeneous discrete structures, such as disordered solids, glasses, fractals, polymer networks and gels, is analytically investigated. It is shown that, while it cannot in general be related to the number of distinct visited points, as in the case of homogeneous lattices, in the case of bounded coordination numbers its asymptotic behaviour at large times can still be expressed in terms of the spectral dimension $\widetilde{d}$, and its exact analytical expression is given. The results show that the asymptotic survival probability is site independent on recurrent structures $(\widetilde{d} \leq 2)$, while on transient structures $(\widetilde{d}>2)$ it can strongly depend on the target position, and such a dependence is explicitly calculated.
\end{abstract}

PACS numbers: 82.33.-z, 82.39.Rt, 05.40.-a 
The kinetics of diffusion limited reactions is deeply affected by geometry and topology, and their description in terms of random walks models is a very powerful tool to explore such a dependence [1, 2]. In some cases, especially on regular lattices, it is possible to establish simple analytical relations between survival probabilities of chemical species and basic random walks functions, making explicit the dependence on universal geometrical parameters such as spatial dimension. The case of irregular structures is more complex, due to the absence of symmetries allowing for a reduction of the degrees of freedom involved in analytical calculations. Therefore, in general, it is not possible to relate time decays and survival probabilities to simple geometrical parameters characterizing the underlying structures.

In this paper, we face a well known kind of reaction, namely the $\mathrm{A}+\mathrm{B} \rightarrow \mathrm{B}$ process, where the A species is immobile, which is known as the target reaction (A is called the target) [3]. This target reaction model is usually introduced to describe a variety of physical, chemical and biochemical processes, such as the Williams-Watts dielectric relaxation in polymers and glasses [4] as well as the poisoning of surface catalysts and of immobilized enzymes [5]. Such a target decay problem has been extensively studied analytically, since it admits exact solutions on homogeneous structures, i.e. on structures where all sites are topologically equivalent [3, 6]. In these cases, it can be shown that the target survival probability decays as a negative exponential of $S(t)$, the mean number of distinct sites visited by a random walk after time t. The case of inhomogeneous structures has been recently investigated for some particular networks, namely Small World Networks (SWN) [7] and Scale Free Networks (SFN) [8], showing that this simple dependence on $S(t)$ no longer holds, and that the deviations from such a behavior are particularly evident at large times. These networks, besides being inhomogeneous, exhibit a peculiar feature: the coordination numbers are not bounded from above in the thermodynamic limit: in other words the maximum coordination number diverges for $N \rightarrow \infty, N$ being the number of sites.

Many interesting real inhomogeneous system, however, don't present such a property and the maximum coordination number is finite even for $N \rightarrow \infty$ : this is the case, for example, of disordered solids, glasses, fractals, polymer networks and gels. All these structures can be mathematically described in terms of "physical graphs" , and the applications of ideas and techniques of algebraic graph theory allows us to obtain analytical results in spite of the lack of invariance and symmetry [9]. In the following we present the mathematical formulation of 
the target decay problem on physical graphs, and we obtain an exact analytical expression for the target survival probability at large times, showing that it can be expressed as a negative exponential of $t$ for transient graphs, and of $t^{\frac{\widetilde{d}}{2}}$ for recurrent graphs, $\widetilde{d}$ being the graph spectral dimension.

Let us begin with some basic definitions [9]:

A graph $\mathcal{G}$ is a countable set $V$ of vertices (or sites) $(i)$ connected pairwise by a set $E$ of unoriented links (or bonds) $(i, j)=(j, i)$.

The graph topology can be algebraically represented introducing its adjacency matrix $A_{i j}$ given by:

$$
A_{i j}=\left\{\begin{array}{l}
1 \text { if }(i, j) \in E \\
0 \text { if }(i, j) \notin E
\end{array}\right.
$$

and the coordination number of site $i$, which is the number of nearest neighbors of $i$, is given by $z_{i}=\sum_{j} A_{i j}$.

The discrete time simple random walk on a graph $\mathcal{G}$ is defined by assuming that at each time step $t$ the walker can only jump to a nearest neighbor site, and that all nearest neighbor sites can be reached with the same probability. Therefore, we can define the jumping probabilities $p_{i j}$ between sites $i$ and $j$ by

$$
p_{i j}=\frac{A_{i j}}{z_{i}}=\left(Z^{-1} A\right)_{i j}
$$

where $Z_{i j}=z_{i} \delta_{i j}$.

Now we introduce the functions $P_{i j}(t)$, each representing the probability of being in site $j$ at time $t$ for a walker starting from site $i$ at time 0 , and the first passage probabilities $F_{i j}(t)$, each representing, for $j \neq i$, the conditional probability for a walker starting from $i$ of reaching for the first time the site $j$ in $t$ steps, and, for $i=j$, the probability of returning to the starting point $i$ for the first time after $t$ steps $\left(F_{i i}(0)=0\right)$.

The fundamental relation between the $P_{i j}(t)$ and the $F_{i j}(t)$ is given by

$$
P_{i j}(t)=\sum_{\tau=0}^{t} F_{i j}(\tau) P_{j j}(t-\tau)+\delta_{i j} \delta_{t 0} .
$$

Introducing the generating functions $\tilde{P}_{i j}(\lambda)$ and $\tilde{F}_{i j}(\lambda)$ by the definition

$$
\tilde{f}(\lambda)=\sum_{t=0}^{\infty} \lambda^{t} f(t)
$$


from eq.(3) we obtain the simpler relation

$$
\tilde{P}_{i j}(\lambda)=\tilde{F}_{i j}(\lambda) \tilde{P}_{j j}(\lambda)+\delta_{i j}
$$

which will be useful in the proof of our main results.

On infinite graphs, representing real systems in the thermodynamic limit, $P_{i i}(t)$ vanishes for $t \rightarrow \infty$. If the graph can be embedded in a finite dimensional Euclidean space, and if the coordination numbers are bounded, i.e. if $\exists z_{\max } \mid z_{i} \leq z_{\max } \forall i \in V$, then $P_{i i}(t)$ vanishes tipically as a power law, whose exponent allows to define the so called (local) spectral dimension $\widetilde{d}$, which is the natural generalization of the Euclidean dimension for dynamical processes $[9]$ :

$$
P_{i i}(t) \sim p_{i} t^{-\widetilde{d} / 2} \quad \text { for } \quad t \rightarrow \infty \quad \forall i \in \mathcal{G}
$$

with $\widetilde{d} \geq 1$, where $p_{i} \equiv p_{0} z_{i}$ (i.e. it depends only on the coordination number of $i$ ) if $\widetilde{d} \leq 2$. For $\widetilde{d} \leq 2, \tilde{F}_{i j}(1)=1$, i.e., the probability of ever reaching any site starting from any site is 1 , and the graph is called recurrent. For $\widetilde{d}>2, \tilde{F}_{i j}(1)<1$, and the graph is called transient.

Now we can define the target decay problem on an infinite graph, following the formalism introduced in [7].

Since each target decays independently of the other ones, we can study the decay of a single target without loss of generality. Let us suppose a target molecule $\mathrm{A}$ is placed at site $k$, while, at time $t=0$, the $\mathrm{B}$ molecules are randomly and independently distributed over the other sites, with average site occupation number $q$. The occupation number distribution at each sites turns out to be Poissonian, and the probability $p(n)$ of finding exactly $n \mathrm{~B}$ molecules at a given site is $p(n)=\frac{q^{n} e^{-q}}{n !}$. For $t>0$, the $\mathrm{B}$ molecules are moving randomly and independently according to the jumping probabilities (2), and the target A is annihilated when it is reached by one of them. Under these hypotheses, it has been shown [7] that the survival probability $\Phi_{k}(t)$ of target $\mathrm{A}$ at time $t$ is given by

$$
\Phi_{k}(t)=e^{-q \Theta_{k}(t)}
$$

where 


$$
\Theta_{k}(t)=\sum_{i \neq k} \sum_{\tau=0}^{t} F_{i k}(\tau)
$$

Now, on homogeneous graphs, $F_{i k}(t)=F_{k i}(t)$, and, due to this symmetry, $\Theta_{k}(t)=$ $S(t)-1$, where $S(t)$ is the number of distinct sites visited by a walker after $t$ steps (on homogeneous graphs it is independent of the starting site $k$ ). This result gives rise to the well known results obtained on $d$-dimensional Euclidean lattices, where, for $t \rightarrow \infty$, $\Theta_{k}(t) \sim \sqrt{t}$ for $d=1, \Theta_{k}(t) \sim t / \ln t$ for $d=2$, and $\Theta_{k}(t) \sim t$ for $d \geq 3[3]$.

On inhomogeneous graphs, $F_{i k}(t) \neq F_{k i}(t)$, and the simple relation mentioned above no longer holds, giving rise to a more complex behavior [7].

Let us proceed to the calculation of the asymptotic behavior of $\Theta_{k}(t)$ by introducing its generating function:

$$
\widetilde{\Theta}_{k}(\lambda)=\sum_{t=0}^{\infty} \lambda^{t} \Theta_{k}(t)=\frac{1}{1-\lambda} \sum_{i \neq k} \tilde{F}_{i k}(\lambda)
$$

From (3), for $i \neq k$, we get $[9]$

$$
\tilde{F}_{i k}(\lambda)=\frac{\tilde{P}_{i k}(\lambda)}{\tilde{P}_{k k}(\lambda)}=\frac{z_{k}}{z_{i}} \frac{\tilde{P}_{k i}(\lambda)}{\tilde{P}_{k k}(\lambda)}
$$

therefore

$$
\frac{z_{k}}{z_{\max }} \frac{\tilde{P}_{k i}(\lambda)}{\tilde{P}_{k k}(\lambda)} \leq \tilde{F}_{i k}(\lambda) \leq \frac{z_{k}}{z_{\min }} \frac{\tilde{P}_{k i}(\lambda)}{\tilde{P}_{k k}(\lambda)}
$$

where $z_{\min } \geq 1$ is the minimum coordination number.

Moreover, since $\sum_{i} P_{k i}(t)=1$ for every $t$, we have that $\sum_{i \neq k} \tilde{P}_{k i}(\lambda)=(1-\lambda)^{-1}-\tilde{P}_{k k}(\lambda)$. Therefore

$$
\frac{z_{k}}{z_{\max }}\left(\frac{1}{(1-\lambda)^{2} \tilde{P}_{k k}(\lambda)}-\frac{1}{1-\lambda}\right) \leq \widetilde{\Theta}_{k}(\lambda) \leq \frac{z_{k}}{z_{\min }}\left(\frac{1}{(1-\lambda)^{2} \tilde{P}_{k k}(\lambda)}-\frac{1}{1-\lambda}\right)
$$

Now we can proceed to the singularity analysis of $\widetilde{\Theta}_{k}(\lambda)$ in order to obtain the asymptotic behavior of $\Theta_{k}(t)$ by applying Tauberian theorems [10].

From (6), we have 


$$
\widetilde{P}_{k k}(\lambda) \underset{\lambda \rightarrow 1^{-}}{\rightarrow} \begin{cases}p_{0} z_{k} \Gamma\left(1-\frac{\widetilde{d}}{2}\right)(1-\lambda)^{\frac{\widetilde{d}}{2}-1} & \text { for } \widetilde{d}<2 \\ p_{0} z_{k} \log (1-\lambda)^{-1} & \text { for } \widetilde{d}=2 \\ \widetilde{P}_{k k}(1) & \text { for } \widetilde{d}>2\end{cases}
$$

therefore, from (12),

$$
\widetilde{\Theta}_{k}(\lambda) \underset{\lambda \rightarrow 1^{-}}{\rightarrow} \begin{cases}\frac{1}{\bar{z}_{k} p_{0} \Gamma\left(1-\frac{\tilde{d}}{2}\right)} \frac{1}{(1-\lambda)^{\frac{\tilde{d}}{2}+1}} & \text { for } \widetilde{d}<2 \\ \frac{1}{\bar{z}_{k} p_{0}} \frac{1}{(1-\lambda)^{2} \log (1-\lambda)^{-1}} & \text { for } \widetilde{d}=2 \\ \frac{z_{k}}{\bar{z}_{k} \widetilde{P}_{k k}(1)} \frac{1}{(1-\lambda)^{2}} & \text { for } \widetilde{d}>2\end{cases}
$$

where $\frac{1}{z_{k}} \equiv \lim _{\lambda \rightarrow 1^{-}}(1-\lambda) \sum_{i} \widetilde{P}_{k i}(\lambda) \frac{1}{z_{i}}=\lim _{\lambda \rightarrow 1^{-}} \sum_{i} \widetilde{P}_{k i}(\lambda) \frac{1}{z_{i}} / \sum_{i} \widetilde{P}_{k i}(\lambda)$, with $z_{\min } \leq \bar{z}_{k} \leq z_{\max }$, is the weighted average of the inverse coordination numbers $1 / z_{i}$, with weights $\widetilde{P}_{k i}(\lambda)$, for $\lambda \rightarrow 1^{-}$. Notice that the hypothesis of boundedness of $z_{i}$ could be replaced with the weaker condition $\bar{z}_{k}<\infty$, leaving our results unchanged. On recurrent graphs, since $\lim _{\lambda \rightarrow 1^{-}} \frac{\tilde{P}_{k i}(\lambda)}{\tilde{P}_{h i}(\lambda)}=1$, uniformly in $i, \bar{z}_{k}$ turns out to be site independent: $\bar{z}_{k}=\bar{z}$. The singularities in $\lambda=1$ in (14) finally give us the following asymptotic behaviors:

$$
\Theta_{k}(t) \underset{t \rightarrow \infty}{\rightarrow} \begin{cases}\frac{\sin \left(\pi \frac{\tilde{d}}{2}\right)}{\bar{z} p_{0} \pi \frac{\tilde{d}}{2}} t & \text { for } \widetilde{d}<2 \\ \frac{1}{\bar{z} p_{0}} \frac{t}{\log t} & \text { for } \widetilde{d}=2 \\ \frac{z_{k}}{\bar{z}_{k} \widetilde{P}_{k k}(1)} t & \text { for } \widetilde{d}>2\end{cases}
$$

These results deserve some comments. First of all notice that, if the graph is homogeneous, we exactly recover the usual asymptotic form of $S(t)$ : this happens not only for Euclidean lattices [3], but also for regular ultrametric spaces [6], and for Bethe lattices, for which the spectral dimension turns out to be infinite [11]. In other cases, such as fractal structures, even if $\Theta_{k}(t)$ is different from $S_{k}(t)$, it turns out to have the same $t$ dependence for $t \rightarrow \infty$.

For completeness sake, we notice that, in some particular cases, it is possible to have logarithmic corrections to the asymptotic behavior described by (6]) [12]: these corrections give rise to logarithmic corrections in (15) too, which are rather simple to calculate; we have neglected them only for simplicity's sake. 
Moreover, for recurrent graphs, the asymptotic behavior of $\Theta_{k}(t)$ is site independent $\left(\Theta_{k}(t) \rightarrow \Theta(t)\right)$ even if the structure is inhomogeneous and the sites are not equivalent. This means that, in spite of inhomogeneity, the recurrent nature of the structure gives rise to asymptotic survival probabilities which are independent of the target position: this is the case, e.g. of many deterministic fractals such as the Sierpinski gasket and the T-fractal [9], and also of recurrent random graphs generated with the constraint of bounded coordination number [13].

On the other hand, when the graph is transient, $\Theta_{k}(t)$ and $\Phi_{k}(t)$ are site-dependent even for $t \rightarrow \infty$. Such a dependence, that only concerns the coefficient of the power of $t$, while the exponent is the same for all sites, is rather intriguing. Indeed, it is a peculiar feature of inhomogeneous transient graphs. In fact, only on these structures the probability $f_{i}$ of ever reaching site $i$, averaged over all possible starting sites, can depend on $i$. In other words, there can exist sites which are more likely to be visited than others, even at large times, and such a property gives rise to the asymptotic site dependence of $\Phi_{i}(t)$.

From the point of view of the applications, the site-dependence is quite interesting, since it means that the target position can affect its survival probabilities at large times, and, in some cases, it is possible to know which sites are more likely to survive (notice that $\widetilde{P}_{k k}(1)$ is the average number of visits to $k$ for a walker starting from $k$ itself). It is particularly noteworthy the case of the so called recurrent on the average transient graphs [14], such as, e.g., $\mathrm{NT}_{D}$ ("Nice Trees of dimension $D$ ") [15], where $\widetilde{P}_{k k}(1)$ is always finite but unbounded from above, i.e., for every $r \in \mathbb{R}$, it exists some $k$ such that $\widetilde{P}_{k k}(1)>r$. In the case of the $\mathrm{NT}_{D}$, which are trees with branches of unbounded length, $\widetilde{P}_{k k}(1)$ is greater for points lying in longer branches: therefore a target placed in such sites has a greater asymptotic survival probability.

The detailed investigation of these aspects is fundamental to design optimal reaction strategies based on geometry, as well as to understand target decay processes in complex biological systems [16].

[1] G. H. Weiss Aspects and Applications of the Random Walks (North-Holland, Amsterdam, 1994). 
[2] D. ben-Avraham and S. Havlin, Diffusion and Reactions in Fractals and Disordered Systems (Cambridge University Press, Cambridge, UK, 2004) .

[3] A. Blumen, G. Zumofen, and J. Klafter, Phys. Rev. B 30, 5379 (1984).

[4] M. F. Shlesinger and E.W. Montroll, Proc. Natl. Acad. Sci. USA 81, 1280 (1984).

[5] J. M. Smith, Chemical Engineering Kinetics (McGraw-Hill-Kogakusha, Tokyo, 1981).

[6] G. Zumofen, A. Blumen, and J. Klafter, J. Chem. Phys. 84, 6679 (1986).

[7] F. Jasch and A. Blumen, Phys. Rev. E 63, 041108 (2001).

[8] M. Galiceanu and A. Blumen, J. Phys. Condens. Matter 19, 065122 (2007).

[9] R. Burioni and D. Cassi, J. Phys. A 38, R45 (2005).

[10] Ph. Flajolet and R. Sedgewick, Analytic Combinatorics (Cambridge University Press, Cambridge, UK, 2009).

[11] D. Cassi, Europhys. Lett. 9, 627 (1989).

[12] D. Cassi and S. Regina, Phys. Rev. Lett. 76, 2914 (1996).

[13] C. Destri and L. Donetti, J. Phys. A 35, 9499 (2002).

[14] D. Cassi, Phys. Rev. Lett. 76, 2941 (1996).

[15] R. Burioni and D. Cassi, Phys. Rev. E 49, R1785 (1994) .

[16] R. E. Marsh, T. A. Riauka, and S. A. McQuarrie, Q. J. Nucl. Med. Mol. Imaging 52, 278 (2008) 